\title{
Hooked: Ethics, the Medical Profession, and the Pharmaceutical Industry
}

\author{
H. Brody \\ Lanham, Maryland: Rowman \& Littlefield Publishers, Inc., 2007, 367 pages, \$27.95
}

What does it mean to you when you read that the pharmaceutical industry has earned nearly two thirds of its profits in the United States given that drug prices in the rest of the industrialized world have become largely governmentcontrolled? Or that continuing medical education has become a big business that amounted to a revenue of $\$ 1.77$ billion in 2003? Or that in 2004 the pharmaceutical industry was reported to have spent $\$ 4.45$ billion on direct advertising to consumers? Or that the American Medical Association received approximately $\$ 54$ million in journal advertising revenues in 1999? Or that professional firms are responsible for ghostwriting a large percentage of published medical articles, especially for multicenter research studies?

To the physician, who has pledged a professional oath to serve the patient who comes in for help and never to take advantage of the patient's vulnerability, some of these figures are disturbing. The moral code of the Hippocratic Oath has its basis in physician-patient trust. A physician has an ethical role to apply the best current scientific knowledge to the individual circumstances of the patient and to practice his healing skills for what is best for the patient. Hooked: Ethics, the Medical Profession, and the Pharmaceutical Industry looks at how modern medicine breaks the trusted physicianpatient relationship by being "hooked" to the pharmaceutical industry, where gifts and rewards influence physicians' decisions and medical institutions are dependent on pharmaceutical support, all in a form likened to addiction. The bond between the physician and the pharmaceutical industry is hard to break. Without the industry and its products, the physician's power to heal vanishes. Physicians no longer gather herbs in the woods or mix potions but instead must work hand in hand with the pharmaceutical industry to serve their patients. In this enlightening book for the medical professional, the author, Dr. Howard Brody, depicts the complex relationship between the medical professional and the pharmaceutical industry. He looks at how this relationship often betrays the public trust, and he offers strategies to shift the dependence of medicine on industry.

As soon as a student enters medical school, the material influence of drug companies is evident. Free lunches, pizza, medical manuals, journal subscriptions, pens, reflex ham-

COPYRIGHT ๑ 2008 by the Society of Nuclear Medicine, Inc. DOI: 10.2967/jnumed.108.055186 mers, penlights, CDs, paperweights, clocks, tote bags, notepads, anatomic models, and mugs, to name just a few items, are given to the soon-to-be doctor. The habit of seeing the drug reps, listening to their advice, and accepting their gifts becomes part of everyday medical practice. The sales reps are attractive, friendly, and likeable. Hugging your drug rep can even be more common than hugging your mother. This type of early pharmaceutical "generosity" creates a trend of selfdeceit that continues throughout the doctor's career. In one interesting study discussed by the author, medical residents were asked if they would consider wearing a small advertising patch on their white coat if the drug companies paid them $\$ 100$ annually. Only $13 \%$ said they would. When the residents were frisked, $97 \%$ of the residents were found to be carrying at least one item with a drug company insignia on it, and the average was 4 items. Fifty-five percent were carrying stethoscope tags with a drug company logo purely for decoration. Contrary to how physicians act, patients when surveyed generally disapprove of pharmaceutical gifts to physicians, especially items that are strictly for personal use. Interestingly, in one of the mentioned surveys, $85 \%$ of medical students disapproved of public officials receiving a $\$ 50$ gift from a prospective bidder, but only $46 \%$ thought it was improper for them to receive the same amount from a drug company.

More disturbingly, Dr. Brody cites that more than $60 \%$ of physicians describe drug reps as having no influence over their prescribing habits. However, study after study shows that hearing about a new drug from a rep and being visited frequently by a rep strongly predict a physician's habit of prescribing the drug in question. Not surprisingly, physicians are blind to the seductive ways of the pharmaceutical industry and come up with a variety of rationalizations, often telling themselves that they can distinguish good sources from bad sources or that drugs reps are an efficient way to stay medically informed.

Continuing medical education has also developed in a direction that is highly agreeable to the pharmaceutical industry. Either the pharmaceutical industry will sponsor a dinner that includes a guest speaker making a presentation favorable to the drug company, or the drug company will work with one of the professional societies organizing a meeting for continuing medical education credit. The pharmaceutical industry will then provide guest speakers, coffee 
and food at breaks, and printed handouts or will help in planning and administration or just set up a display booth. Stemming from a culture of entitlement based on the medicine-industry relationship, physicians attending the professional society's meetings come to expect low registration fees, luxurious accommodations, and free dinner and entertainment each night. If societies were to hold their meetings in less accommodating means, attendance by physicians would be expected to drop.

The quality of research produced in the United States as a result of the medicine-industry link is of great concern to the author. Drug companies provide important funds for research but may also manipulate the data into favorable results. One alarming practice that is mentioned by the author is ghostwriting of scientific articles. As one example, a firm called Current Medical Directions coordinated 87 articles on the antidepressant Zoloft, with 55 articles being published in top medical journals such as New England Journal of Medicine and JAMA. Based on contact with the authors of these studies and other sources, in only 5 articles did the listed authors have access to the raw data. For most of the articles, the authors' names were added only after Current Medical Directions finished writing the articles, although final editing was allowed by the authors. This type of questionable practice, among others cited in the book, really undermines the quality and validity of medical research and journals.

Dr. Howard Brody has written a powerful book that is relevant to all our practices and questions the relationship between medicine and the pharmaceutical industry. Laws and regulations may be enacted to control this relationship; however, the medical profession, not politicians, is ultimately responsible for its own integrity.

Robert Matthews

Stony Brook University Hospital Stony Brook, New York 